\title{
A Case of Nivolumab-Induced Cutaneous Toxicity with Multiple Morphologies
}

\author{
Emily D. Nguyen ${ }^{a} \quad$ Yun K. Xue ${ }^{a, b} \quad$ Melissa Danesh ${ }^{a, b} \quad$ Amir Ameri $^{a}$ \\ Christina Q. Weng ${ }^{a, b}$ Nikolai Klebanov $^{a}$ Ruth K. Foreman ${ }^{c}$ \\ Rosalynn M. Nazarian ${ }^{c}$ Shadmehr Demehria Hensin Tsao ${ }^{a}$ \\ Daniela Kroshinsky a \\ a Department of Dermatology, Massachusetts General Hospital, Boston, MA, USA; \\ ${ }^{b}$ Harvard Combined Dermatology Residency Training Program, Boston, MA, USA; \\ 'Department of Dermatopathology, Massachusetts General Hospital, Boston, MA, USA
}

\section{Keywords}

Nivolumab · Immune checkpoint inhibitor · Cutaneous toxicity · Lichenoid reaction

\begin{abstract}
Cutaneous reactions are among the most prevalent immune-related adverse events in patients treated with immunotherapy. Given that immunotherapies often act through blocking inhibitory signals on T cells, these treatments also have the potential to generate a host of immune toxicities. We report the case of a 73-year-old woman with a history of non-small cell lung cancer treated with nivolumab 10 months prior to presentation who developed painful nodules, bullae, and a scaly rash on her extremities. Four months after discontinuation of nivolumab, she noted an acute eruption of painful nodules on her extremities, followed by pink papules and tense bullae on her palms and soles. Biopsies were performed of three lesions in sites of varying morphologies. These findings were felt to be consistent with a nivolumab-induced lichenoid reaction. She was initially treated with intralesional steroid injections, topical steroid ointment, and liquid nitrogen cryotherapy with minimal improvement. As the lesions continued to progress, the patient was admitted to the hospital and started on intravenous methylprednisolone. She eventually transitioned to daily oral prednisone with a slow taper with good effect and no recurrence of lesions.

(c) 2020 The Author(s)

Published by S. Karger AG, Basel
\end{abstract}

E.D.N. and Y.K.X. are co-first authors in the preparation of the manuscript. 
Nivolumab, the first monoclonal antibody against the immune checkpoint inhibitor programmed cell death protein-1 (PD-1), is approved for clinical use for the treatment of advanced melanoma and metastatic non-small cell lung cancer. Given their mechanism, these treatments also have the potential to generate a host of immune toxicities, otherwise known as immune-related adverse events (irAEs). Dermatologic toxicities are amongst one of the most prevalent irAEs, seen in approximately a third of all patients treated with immunotherapies [1]. We report the case of a 74-year-old woman with a history of non-small cell lung cancer treated with nivolumab 10 months prior to presentation who developed painful nodules, bullae, and a scaly rash on her extremities.

\section{Case Report}

A 74-year old woman presented with non-small cell lung cancer treated initially by wedge resection, chemotherapy, and radiation. Nivolumab was initiated after a subsequent metastasis to the left lower lobe and mediastinal lymph nodes. Her treatment course was complicated by thyroiditis and large oral and vaginal ulcers, leading to discontinuation of nivolumab. Treatment with doxycycline led to the resolution of the patient's mucosal ulcerations. Four months later, the patient noted an acute eruption consisting of painful, friable pruritic nodules on her extremities. Over the course of several weeks, she developed lesions of varying morphologies: multiple bright pink papules with a white peripheral border, large hyperkeratotic nodules and plaques, some with central ulceration, and several tense bullae along bilateral palms and soles (Fig. 1). Several hyperkeratotic lesions were treated with liquid nitrogen cryotherapy and intralesional triamcinolone with minimal effect.

Biopsies were performed of lesions of varying morphologies. Histopathologic examination of the hyperkeratotic lesions revealed endophytic squamous proliferation with a lichenoid inflammatory infiltrate, consistent with hypertrophic lichen planus (LP). The buccal mucosa biopsy revealed ulcerated squamous mucosa with dense lichenoid lymphoplasmacytic infiltrate. Biopsy of a vesicular lesion revealed subepithelial vesicle with associated epidermal hyperplasia, lichenoid interface change, and perivascular lymphocytic and neutrophilic infiltrate with pigment incontinence (Fig. 2), consistent with lichenoid hypersensitivity reaction, bullous LP, or bullous pemphigoid. Direct immunofluorescence examination was negative for IgG and IgM reactivity along the basement membrane zone. These findings were felt to be consistent with a nivolumab-induced lichenoid reaction.

The patient was started on intravenous methylprednisolone $60 \mathrm{mg}$ twice daily, which was eventually transitioned to oral prednisone $80 \mathrm{mg}$ daily on discharge. She continued to receive wound care with topical clobetasol $0.05 \%$ ointment and non-adherent bandages, and her pain was well-controlled with hydromorphone. Two weeks after discharge, she showed significant improvement in pain and decrease in size and number of hyperkeratotic papules and plaques. She was eventually trialed to acitretin $10 \mathrm{mg}$ every other day for possible flare prevention but discontinued due to nausea.

\section{Discussion}

IrAEs in the context of immune checkpoint inhibitors are driven by blockade of T-cell suppression and modulation of immunosurveillance [2-4]. Blockade of the programmed cell death receptor on activated $\mathrm{T}$ cells leads to an overall increased inflammatory response to antigens and tumors, thus shifting the balance to an anti-tumor response [3]. Dermatologic irAEs typically occur within days or weeks of treatment, though onset may be delayed, 

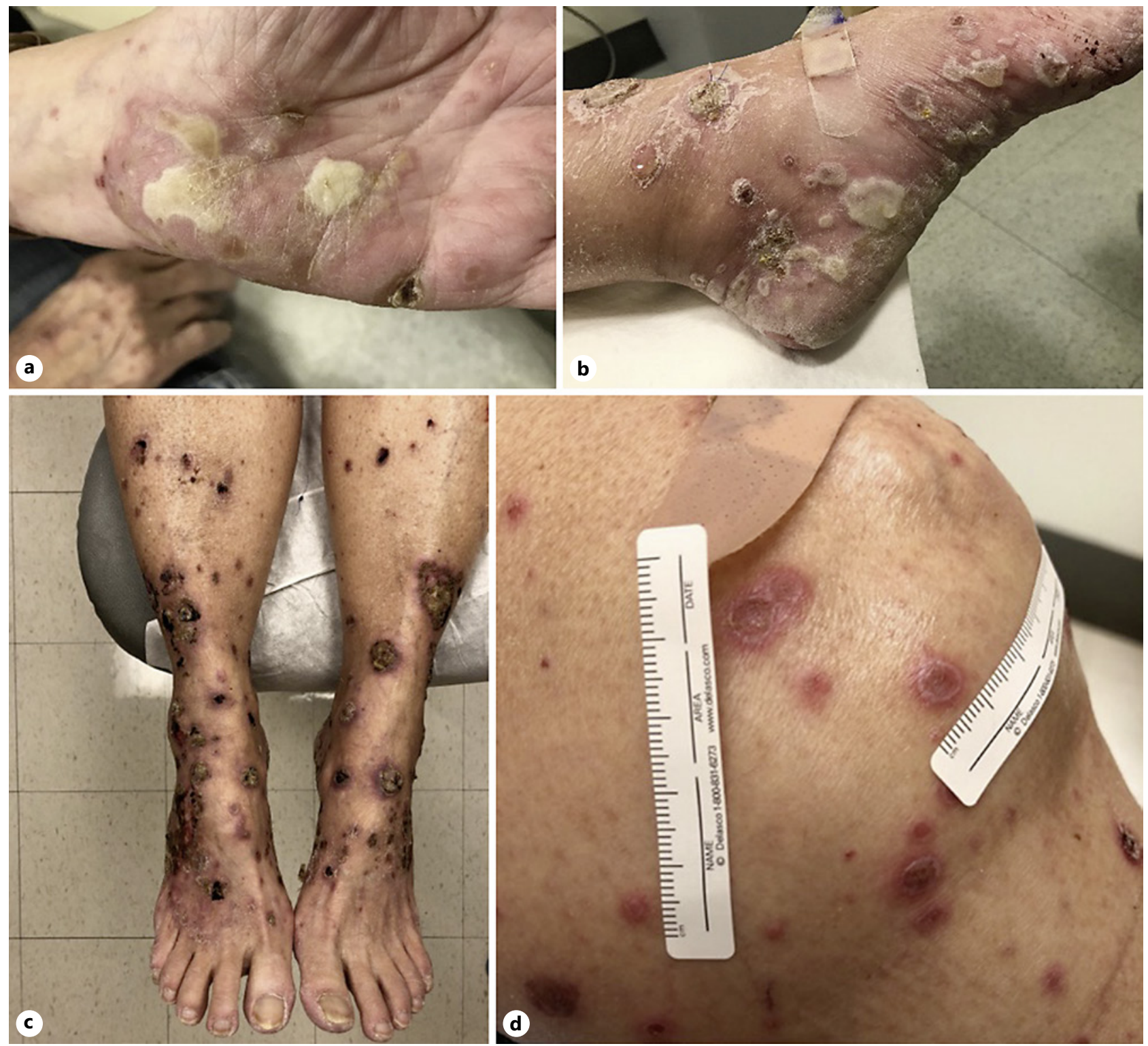

Fig. 1. Different clinical morphologies. Tense bullous lesions on palms (a) and soles (b). c Large hyperkeratotic nodules and plaques, some with central ulceration. d Pink flat papules with white peripheral border, some with scale.

appearing 3-6 months after initiating the anti-PD-1 agent [5]. A delayed effect of immune checkpoint antibodies, as seen in our patient, can also occur sometimes up to 1 year after the initiation of anti-PD-1 treatment, so keeping a high clinical suspicion for anti-PD-1 cutaneous toxicity is essential [6]. To our knowledge, the concomitant manifestations of lichenoid processes, including LP-like lesions, keratoacanthomas, and bullous LP, in an individual patient have not been reported.

A case series of three patients receiving pembrolizumab for metastatic melanoma described the development of erythematous to violaceous papules and plaques 7-9 weeks after drug initiation. A single-institution cohort study of 82 patients on single-agent anti-PD-1 therapy for metastatic melanoma identified lichenoid reaction development in 14 patients. 

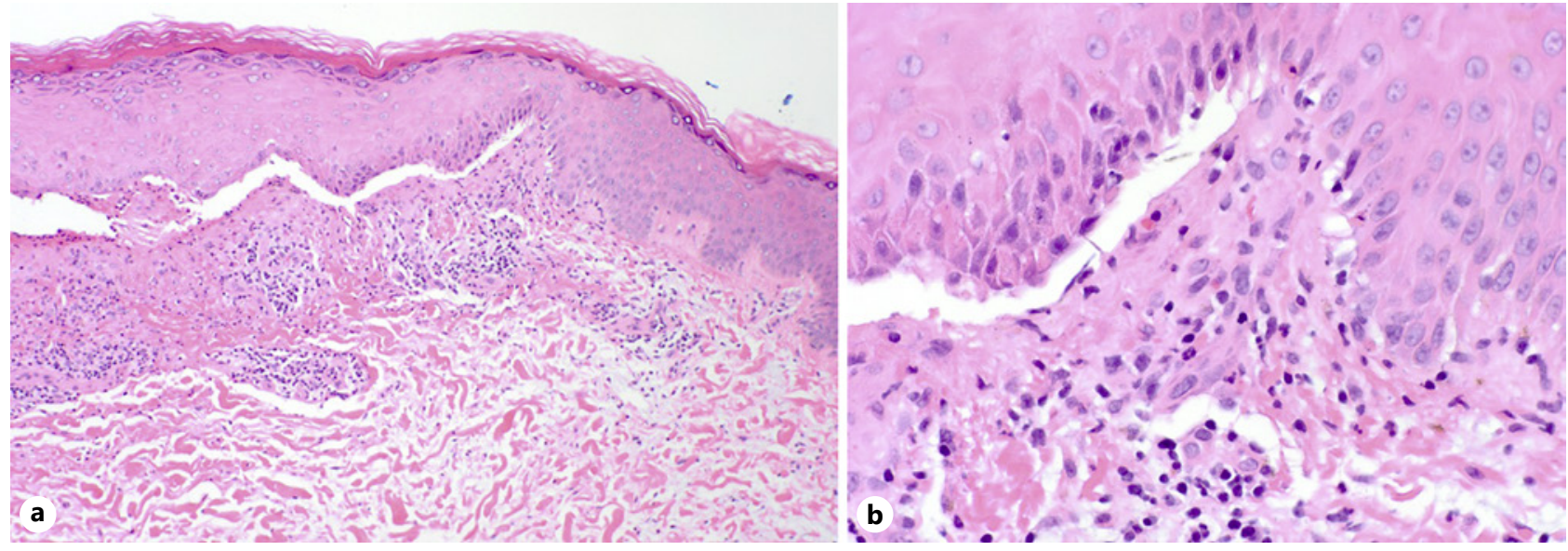

Fig. 2. Left medial ankle biopsy. Epidermal hyperplasia with lichenoid inflammatory infiltrate and subepidermal bulla. a Low-power view (hematoxylin and eosin, $\times 100$ ). b High-power view (hematoxylin and eosin, $\times 400)$.

Of these patients, $17 \%$ developed lichenoid reactions, 17\% developed eczema, and 15\% developed vitiligo [7]. In addition, two patients developed all 3 cutaneous toxicities, and 9 developed certain combinations of all three events [7].

Our patient also developed hypertrophic nodules on a background of lichenoid drug reaction. A recent case series of 3 patients describes pembrolizumab-associated eruptive keratoacathomas that resolved with monthly intralesional steroid injections $(20 \mathrm{mg} / \mathrm{mL})$, adjuvant topical treatment every other day, and liquid nitrogen cryotherapy [8]. In this series, all patients achieved complete response after 1 month of treatment. While our patient reported minimal improvement in her hands one week after the initial $20 \mathrm{mg} / \mathrm{mL}$ steroid injections and cryotherapy, these treatments were stopped when she developed bullous lesions. It is possible that continued serial treatments would have led to resolution of these lesions.

Finally, our patient additionally had bullous lesions on examination. A number of reports have recently reported PD1-inhibitor-induced bullous pemphigoid [9-13]. At a single institution, bullous eruptions were seen in $9(1 \%)$ of 853 patients treated with anti-PD1 therapy over a period of 2 years. In a case series of 5 patients with bullous lesions, 4 were found to have bullous pemphigoid and were successfully treated with systemic and topical steroids [12]. The negative direct immunofluorescence in our patient indicates that the bullae represented a component of the lichenoid drug reaction.

\section{Conclusion}

Although each individual morphology has been reported in the setting of PD-1 inhibitor therapy, we report the first case of LP-like lesions, keratoacanthomas, and bullous LP morphologies arising concomitantly after nivolumab treatment. The inflammatory infiltrate on biopsy and their complete response to intravenous corticosteroids suggest that they represent irAE to nivolumab. Patients receiving nivolumab will need to be closely monitored for occurrence of irAEs. Further research is necessary to understand the immunologic mechanism underlying nivolumab-induced cutaneous toxicity. 


\section{Statement of Ethics}

The patient gave consent to write up her case for publication. Specific and identifiable information has been avoided in discussion of this case.

\section{Disclosure Statement}

The authors have no conflicts of interest to declare.

\section{Funding Sources}

No funding sources.

\section{Author Contributions}

All authors take responsibility for the integrity and accuracy of the manuscript and approved the final version of the manuscript.

\section{References}

1 Maughan BL, Bailey E, Gill DM, Agarwal N. Incidence of Immune-Related Adverse Events with Program Death Receptor-1- and Program Death Receptor-1 Ligand-Directed Therapies in Genitourinary Cancers. Front Oncol. 2017 Apr;7(56):56.

2 Yoest JM. Clinical features, predictive correlates, and pathophysiology of immune-related adverse events in immune checkpoint inhibitor treatments in cancer: a short review. ImmunoTargets Ther. 2017 Oct;6:73-82.

3 Alsaab HO, Sau S, Alzhrani R, Tatiparti K, Bhise K, Kashaw SK, et al. PD-1 and PD-L1 Checkpoint Signaling Inhibition for Cancer Immunotherapy: Mechanism, Combinations, and Clinical Outcome. Front Pharmacol. 2017 Aug;8:561-561.

4 Topalian SL, Sznol M, McDermott DF, Kluger HM, Carvajal RD, Sharfman WH, et al. Survival, durable tumor remission, and long-term safety in patients with advanced melanoma receiving nivolumab. J Clin Oncol. 2014 Apr;32(10):1020-30.

5 Robert C, Ribas A, Wolchok JD, Hodi FS, Hamid O, Kefford R, et al. Anti-programmed-death-receptor-1 treatment with pembrolizumab in ipilimumab-refractory advanced melanoma: a randomised dosecomparison cohort of a phase 1 trial. Lancet. 2014 Sep;384(9948):1109-17.

6 Belum VR, Benhuri B, Postow MA, Hellmann MD, Lesokhin AM, Segal NH, et al. Characterisation and management of dermatologic adverse events to agents targeting the PD-1 receptor. Eur J Cancer. 2016 Jun;60:12-25.

7 Hwang SI, Carlos G, Wakade D, Byth K, Kong BY, Chou S, et al. Cutaneous adverse events (AEs) of antiprogrammed cell death (PD)-1 therapy in patients with metastatic melanoma: A single-institution cohort. J Am Acad Dermatol. 2016 Mar;74(3):455-61.e1.

8 Freites-Martinez A, Kwong BY, Rieger KE, Coit DG, Colevas AD, Lacouture ME. Eruptive Keratoacanthomas Associated With Pembrolizumab Therapy. JAMA Dermatol. 2017 Jul;153(7):694-7.

9 Lopez AT, Geskin L. A Case of Nivolumab-Induced Bullous Pemphigoid: Review of Dermatologic Toxicity Associated with Programmed Cell Death Protein-1/Programmed Death Ligand-1 Inhibitors and Recommendations for Diagnosis and Management. Oncologist. 2018 Oct;23(10):1119-26.

10 Siegel J, Totonchy M, Damsky W, Berk-Krauss J, Castiglione F Jr, Sznol M, et al. Bullous disorders associated with anti-PD-1 and anti-PD-L1 therapy: A retrospective analysis evaluating the clinical and histopathologic features, frequency, and impact on cancer therapy. J Am Acad Dermatol. 2018 Dec;79(6):1081-8.

11 Mochel MC, Ming ME, Imadojemu S, Gangadhar TC, Schuchter LM, Elenitsas R, et al. Cutaneous autoimmune effects in the setting of therapeutic immune checkpoint inhibition for metastatic melanoma. J Cutan Pathol. 2016 Sep;43(9):787-91.

12 Jour G, Glitza IC, Ellis RM, Torres-Cabala CA, Tetzlaff MT, Li JY, et al. Autoimmune dermatologic toxicities from immune checkpoint blockade with anti-PD-1 antibody therapy: a report on bullous skin eruptions. J Cutan Pathol. 2016 Aug;43(8):688-96.

13 Kaunitz GJ, Loss M, Rizvi H, Ravi S, Cuda JD, Bleich KB, et al. Cutaneous Eruptions in Patients Receiving Immune Checkpoint Blockade: Clinicopathologic Analysis of the Nonlichenoid Histologic Pattern. Am J Surg Pathol. 2017 Oct;41(10):1381-9. 